\title{
1 Plant-pollinator specialization: Origin and measurement of
}

\section{2 curvature}

3 Author Names: Mannfred M.A. Boehm ${ }^{1,3, *}$, Jill E. Jankowski ${ }^{2,3}$, Quentin C.B. Cronk ${ }^{1,3}$

$4 \quad{ }^{*}$ Corresponding Author: mannfred.boehm@ubc.ca

5 Author affiliations: ${ }^{1}$ Department of Botany, University of British Columbia, 3156-6270

6 University Boulevard, Vancouver, BC, Canada, V6T 1 Z4.

$7 \quad{ }^{2}$ Department of Zoology, University of British Columbia, 4200-6270 University Boulevard,

8 Vancouver, BC, Canada, V6T 1 Z4.

$9{ }^{3}$ Biodiversity Research Centre, University of British Columbia, 2212 Main Mall, Vancouver,

10 BC, Canada, V6T 1 Z4.

11 Submission Type: Synthesis and Perspective

12 Abstract: 198 Words

13 Main Body: 5900 Words

14 Figures: 5 (colour). Additional figures in Supp. Mat.

15 Tables: 1

16 Keywords: hummingbird, pollination, curvature, morphometerics, floral diversity 


\section{Abstract}

19 A feature of biodiversity is the abundance of curves displayed by organs and organisms.

20 Curvature is a widespread, convergent trait that has important ecological and evolutionary

21 implications. In pollination ecology, the curvature of flowers and pollinator mouthparts

22 (e.g. hummingbird bills) along the dorsiventral plane has been associated with specialization,

23 competition, and species co-existence. Six methods have historically been used to measure

24 curvature in pollination systems; we provide a solution to this inconsistency by defining

25 curvature using well-established concepts from differential geometry. Intuitively, curvature is the

26 degree to which a line is not straight, but more formally, it is the rate at which the tangent of a

27 curve changes direction with respect to arc length. Here, we establish a protocol wherein a line is

28 fitted against landmarks placed on an image of a curved organ or organism, then curvature is

29 computed at many points along the fitted line and the sum taken. The protocol is demonstrated

30 by studying the development of nectar spur curvature in the flowering plant genus Epimedium

31 (Berberidaceae). By clarifying the definition of curvature, our aim is to make the language of

32 comparative morphology more precise and broadly applicable to capture other curved structures

33 in nature. 


\section{The ecology of flower-pollinator curvature}

"We are beginning to understand why some hummingbird bills are long, whereas others are short, and why some hummingbird flowers are wide, whereas others are narrow. Now, why are bills of some hummingbirds and the tubes of the flowers they visit curved?" - Temeles (1996).

At the center of plant-pollinator diversification is a remarkable variety of floral form. The notion that plant communities experience selection to reduce interspecific mating ("floral isolation," Grant, 1949) points to the importance of floral diversity in initiating and reinforcing reproductive isolation (Armbruster and Muchhala, 2009). For example, patterns of character displacement in sympatric Centropogon C.Presl (Campanulaceae) suggest that competition for pollinators led to the divergence of floral traits associated with bat and hummingbird pollination (Lagomarsino and Muchhala, 2019). In the case of South African Lapeirousia Pourr. (Iridaceae), geographic variation in floral tube length has subsequently initiated reproductive isolation between morphs with short and long corolla tubes, despite sharing the same fly pollinator (Minnaar et al., 2019). While patterns of plant-pollinator evolution point to both contemporaneous and asymmetrical coadaptation (Cardinal and Danforth, 2013; Tripp and McDade, 2013), floral morphology can both be the cause and result of plant-pollinator diversification (Kay and Sargent, 2009; Niet and Johnson, 2012; Ollerton, 2017).

Flower-pollinator curvature as viewed from the side (dorsiventral plane) has been a trait of special interest throughout the post-Darwin era of pollination ecology. However, some floral curvature has origins that may precede any particular ecological function. Instead, curved flower parts (e.g. nectar spurs) might develop within buds when constrained for space. That is, during 
57 bud development nectar spurs elongate and curve when met with resistance from the enclosing

58 bud tissue. Following bud opening flower parts may straighten but retain some degree of

59 curvature at maturity. While curvature may thus originate from a developmental constraint or

60 lack of selection for straightness, there can be positive selection for curvature when it increases

61 the complexity of nectar extraction and mechanical interaction between the anthers and the body

62 of the pollinator (Young, 2008; Collins, 2008).

63 One consequence of floral curvature is pollinator partitioning. In making pollinator

64 observations of the Cape flora, Scott-Elliott (1890) noticed that the flowers of Leonotis

65 ocymifolia (Burm.f.) Iwarsson (Lamiaceae) visited by sunbirds (Nectariniidae) were "curved

66 with the same curvature as that of the bird's beak." (p. 272). Robertson (1889) insightfully notes

67 that the curved nectar spur of Viola L. (Violaceae) "serves to limit the insect visits much more

68 than the mere length of the spur." (p. 172). Stiles (1975) first posited that Neotropical Heliconia

69 L. (Zingerberales) partition hummingbird (Trochilidae) visitation by flower and bill curvature,

70 and that specialization by curve-billed hummingbirds allow co-existence within this species-rich

71 clade. Subsequent research supports this hypothesis (Maglianesi et al., 2014): along the slopes of

72 the Central Cordillera of Costa Rica, the degree of flower and hummingbird bill curvature is

73 proportional to plant-pollinator interaction strength (sensu Dehling et al., 2014) and extent of

74 specialization (sensu Blüthgen et al., 2006). In addition to interspecific partitioning of resources,

75 many hummingbirds exhibit sexual dimorphism in bill shape: females have greater bill curvature

76 than males and forage for nectar from curved flowers (Temeles et al., 2005, 2010). Explanations

77 for this pattern range from reduced competition for nectar resources between sexes (Paton and

78 Collins, 1989; Temeles et al., 2010) to selection for mechanically superior bill shapes during

79 male-male competition for territory (Rico-Guevara and Araya-Salas, 2014). Even from the 
80 earliest observations, curvature has been synonymous with specialization; we expect curvature to

81 limit the range of functional taxa in a plant-pollinator mutualism and strengthen interactions

82 between the existing participants.

83 More recently the scope of plant-hummingbird research has expanded to address the

84 biogeography of curvature. As predicted by Stiles (2004), Maglianesi (2015a) and Sonne (2019)

85 found plant-hummingbird curvature to be more represented across species in the lowlands of the

86 Neotropics compared to higher elevations. In this case, plant-pollinator curvature is a form of

87 niche divergence evolving in species-rich lowland habitats, where species experience relatively

88 higher competition (e.g. for nectar or pollen vectors) than in the adjacent Andes mountains

89 (Stiles, 2004; Graham et al., 2009). Furthermore, because plant and hummingbird morphology is

90 better matched (i.e. more specialized) at lower latitudes (Sonne et al., 2020) and hummingbirds

91 with curved bills are predominately tropical, we might expect the occurence of curvature in these

92 taxa to have a predictable latitudinal distribution.

93 While curvature mediates specialization in plant-hummingbird systems, in other

94 nectivorous bird groups curvature appears to evolve in the absence of selection for resource

95 partioning. In plant-passerine systems, curvature is more prevalent in pollinators than in flowers.

96 Straight flowers do not necessarily exclude pollination by curve-billed birds; for example, the

97 straight, tubular flowers of African Aloe L. (Asphodelaceae) are pollinated by curve-billed

98 sunbirds (Paton and Collins, 1989), and the small campanulate flowers of Vaccinium L.

99 (Ericaceae) are pollinated by the Hawaiian honeycreeper Drepanis coccinea (Fringillidae,

100 Carothers, 1982). For these passerine clades, a dietary shift to nectivory may drive the evolution

101 of bill curvature because (ancestral) insect-pollinated plants require perching at angles not 
102 directly facing the flower opening (Paton and Collins, 1989). Furthermore, probing concealed

103 nectar from a fixed perch is an inherently arc-like motion (analogous to reaching into a tall cup

104 to extract, say, an ice cube). Recent experimental work found that Amethyst sunbirds

105 (Chalcomitra amethystina) extract nectar more efficiently when flowers are curved towards a

106 perch, indicating that bill curvature may evolve from the inherent arc-like motion of probing

107 tubular flowers (Johnson et al., 2020).

109 (Africa, Australasia), Hawaiian honeycreepers, and hummingbirds (Nearctic, Neotropic)

110 suggests that bill curvature is widespread in the passerine families but is generally restricted to a

111 subfamily of hummingbirds (Phaethornithinae, Paton and Collins, 1989). While hummingbirds

112 possess unique musculature and wing shape for hovering and manoeuvring adeptly while feeding

113 (Dakin et al., 2018), passerines typically perch to probe for nectar. For plant-passerine systems,

114 primarily insectivorous birds may have evolved curved bills to feed at awkward angles from

115 plants with straight, tubular flowers, and only in some cases does reciprocal adaptation produce

116 curved flowers, e.g. sunbird-pollinated Streptocarpus dunnii Mast. (Gesneriaceae, Hughes et al.,

117 2007). The evolution of reciprocal curvature in such plants may be driven by selection to either

118 exclude inefficient pollinators such as bees, which otherwise would compete for nectar and

119 pollen, or to increase the precision of pollen placement. It is worth noting that in landbirds, the

120 evolution of bill shape is coupled with skull shape (Bright et al., 2016; Navalón et al., 2020).

121 Therefore, for some nectivorous passerines the evolution of bill shape is influenced not only by

122 flower morphology, but also the biomechanical factors that restrict skull shape (Navalón et al.,

123 2020). Thus, unlike plant-hummingbird systems (in which curvature mediates pollinator 
124 partitioning), selection for curvature in plant-passerine systems appears to operate on nectivorous

125 birds moreso than the plants they pollinate.

126 Floral diversity contributes to floral isolation and diversification in the angiosperms (Kay

127 and Sargent, 2009; Armbruster and Muchhala, 2009; Vamosi et al., 2018). Similarly, dietary

128 specialization within pollinator clades has contributed to the diversification of mouthpart

129 morphology (Weinstein and Graham, 2017; Maruyama et al., 2018). In both cases, curvature is a

130 widespread feature of morphological diversity. Therefore, to synthesize our knowledge of curved

131 plant-pollinator systems, curvature is a concept that needs an exact definition and method of

132 measurement. In the following section we summarize the approaches used in measuring

133 curvature within the field of pollination ecology, and evaluate the strengths and shortcomings of

134 each. Building on this assessment, we offer a conceptualization of curvature that improves the

135 precision of measurement of this trait. Although this review is motivated by the problem of

136 measuring curvature in plant-pollinator systems, the solution is general to any biological form

137 modelled as a line curve: we then apply this method to floral curvature in an example

138 demonstration. 
140 Summary of the literature: history of measuring curvature in pollination

\section{1 ecology}

142 We searched the scientific literature for studies of floral or pollinator mouthpart curvature, as

143 these traits are commonly measured as a proxy for specialization. We make the distinction

144 between measuring curvature in a single plane (e.g. the dorsiventral plane of flowers) versus the

145 curvature of surfaces. While single plane images are analysed for line-curvature, measurements

146 of specimens measured in two planes (e.g. dorsiventral and transverse) can be used to analyse

147 surface (Gaussian) curvature (Nath et al., 2003; Coen and Rebocho, 2016). At present, surface

148 curvature has yet to be considered in the context of pollination, and is probably of limited

149 importance. Floral tubes are generally circular in cross-section and surface curvature takes the

150 form of simple or flared cylinders, and complex and mathematically interesting curved surfaces

151 such as saddle-shapes, are absent. Furthermore, because line and surface curvature are related

152 mathematical concepts, it will benefit pollination research to clarify the simplest case (lines),

153 with the goal of generating interest in related ideas, including the curvature of surfaces.

154 The literature was sourced by querying Web of Science and Google Scholar for a topic

155 search of (curv*) AND (pollinat*) AND (flower OR corolla OR *bird OR *bee OR moth OR

$156 *$ *ly). The initial search returned over 300 studies that were then screened for those that

157 measured curvature of floral organs (e.g. petals, styles) and/or animal mouthparts (e.g. bird bills,

158 moth tongues). We sorted studies based on the criteria that 1) the study focused on petal

159 curvature or animal pollination, including qualitative measures of curvature or 2) the study

160 measured curvature of a floral organ other than petals (e.g. style curvature in autogamous

161 species) or animal mouthparts outside of a pollination context (e.g. taxonomic classification). 
162 Under the first criterion, 45 studies were identified to have used some form of curvature metric

163 (Table 1). An additional 17 studies (second criterion) are included in Table S1. There were

164 numerous studies of plant-animal morphology that did not address curvature - these were

165 omitted from our analysis.

166 In our survey, the dedicated discussion of dorsiventral curvature in plant-pollinator

167 interactions begins with Hainsworth (1973), in reference to Heliconia and hermit hummingbirds.

168 Curvature in pollination ecology is first empirically studied by Gill and Wolf (1978), though

169 methods for measuring curvature of bird appendages outside of a pollination context can be

170 found much earlier (Baldwin et al., 1931). We identified six common approaches to measuring

171 curvature in pollination systems. These are:

172 1. qualitative description, e.g. "very curved," "less curved," but these are generally no longer

173 used;

174 2. the arc:chord method defines curvature as a ratio of two lines: an arc fitted to the curve of a

175 flower or mouthpart (e.g. bird bill) from its tip to base, and the straight line (chord)

$176 \quad$ subtending the arc (Figure 1);

177 3. the mandibular index defines curvature as a ratio of two lines: a straight line from base to

178 tip (chord) and a perpendicular line that measures the maximum height of the flower/bill arc

$179 \quad$ (versine).

180 4. the angle of deflection method defines curvature as the angle between the tangent line at the

181 base of the flower/bill and the straight line from base to tip (chord);

182 5. the inverse radius method which approximates the arc of the flower/bill as a segment of a

183 circle. Curvature is defined as the inverse radius of the fitted circle; 
184 6. geometric morphometrics, which quantifies shape as a configuration of homologous points (landmarks) existing on a coordinate plane (Figure S2). can be taken in the field or determined easily from photographs. The methods are intuitive and in the simplest case, require only a ruler, string, and protractor. Temeles (2009) pointed out that, for curves well fitted by a circle, the inverse radius method is interchangeable with the angle of deflection method because the radius can be calculated from the length and angle of a chord

191 (tangent chord theorem, Bell, 1956). Similarly, for circles, arc length and versine can be 192 computed from the radius and angle of deflection (Zwillinger, 2018). Given the required

193 additional geometric information, for curves well fitted by a circular arc, methods $2-4$ will be 194 proportional to the inverse radius. In fitting a curve with a circle we assume that curvature is constant across the specimen.

196 However, when curvature deviates from constant, methods 2-5 are less suitable (discussed in 197 Berns and Adams, 2010). The angle of deflection and mandibular index are computed from only 198 two or three landmarks, respectively. Any changes in curvature between landmarks are not 199 considered - this is problematic when curvature varies across the specimen (e.g. a hook at the 200 end of a bird bill). The inverse radius method assumes the curve to have constant curvature. The 201 arc:chord method, because it considers arc length, implicitly contains information about 202 curvature across the entire specimen - for a curved line in a plane, arc length and total curvature 203 are proportional (Section 3). However, the curvature of a local feature cannot be extracted given 204 only the length of the arc and chord. Overall, while these methods succeed in estimating the total 205 curvature of an entire curve, they are not designed to account for fluctuating curvature caused by 206 locally curved features. 
An additional problem is a lack of consensus terminology and methodology. For example,

208 the arc:chord method is also called the maxillary index, while the angle of deflection method is

209 sometimes referred to as the angle of declension method. Many studies create their own

210 terminology for the concept of arc length: the length of a curve between two points. Most studies

211 define their own terms for measuring and reporting curvature without reference to previous

212 studies. We found no discussion of units or of their meaning. This creates uncertainty about how

213 to compare and convert metrics used between studies. We also found no discussion or methods

214 accounting for allometry. We believe these problems could be remedied by referring to the

215 mathematical literature for the derivation and definition of curvature and related concepts (see:

216 Section 3).

217 Starting with Berns and Adams (2010) geometric morphometrics (GM) emerges in the

218 pollination literature. GM is broadly concerned with the covariance of shape and associated or

219 causal variables (Bookstein, 1991; MacLeod, 2002), for example, how flower shape might

220 covary with local pollinator communities (Gómez et al., 2009). This approach has steadily

221 gained in popularity due to its mathematical rigour, reproducibility, and the appealing visual

222 representations of shape variation (Olsen, 2017). Additionally, because GM has a traceable

223 mathematical lineage (Bookstein, 1991), its vernacular is well-defined and used consistently

224 between practitioners. Here, we highlight some of the most important features of GM to

225 introduce relevant concepts, and recommend the concise and authoritative introduction by

226 Webster and Sheets (2010) for more details.

228 the primary data used to quantify shape. Methods 2-5 (above) fit into this category. Typically 
these measurements are anchored by landmarks_-toplogically or biologically homologous points

230 that can be located on all specimens (detailed in Bookstein, 1991; MacLeod, 1999). In geometric

231 morphometrics landmarks are assigned across the specimen, with the goal of representing its

232 shape as completely as possible. A typical protocol for a 2-D object begins by placing the

233 specimens on an $x y$-grid and assigning $x y$-coordinates to landmarks (Figure $\mathrm{S} 2$ ). In a

234 comparative study, the samples are overlaid so that their shape information is isolated from their

235 orientation, location, and size. This is done using a least-squares type protocol, most commonly

236 the Generalized Procrustes Analysis (Rohlf and Slice, 1990). In outline-based GM, the $x y$

237 coordinates of landmarks are fitted by form or shape functions (sensu MacLeod, 2012) and

238 decomposed by elliptic (Kuhl and Giardina, 1982) or 'ZR' (Zahn and Roskies, 1972) Fourier

239 analysis, respectively. Harmonic shape variables from a Fourier analysis are then used to

240 calculate the principal components of shape variation (MacLeod, 2012). In landmark-based GM,

241 the set of landmarks summarising the shape of an organism is treated as a 'landmark

242 configuration.' Configurations exist in a shape space defined by the number of landmarks and

243 spatial dimensions implemented. These configurations are then projected onto a simpler

244 Euclidian space, analogous to the reduction of a spherical Earth onto a two-dimensional map

245 (Webster and Sheets, 2010). From here, familiar statistical procedures (e.g. PCA) can be

246 performed to quantify variation in landmark configurations (shape) between samples.

248 or end-member deformation grids (Bookstein, 1991) at both ends of a given principal axis. This

249 enables a qualitative description of the primary trait(s) varying along said axis. For example,

250 visual inspection of deformation grids along PC1 of Figure 4 (Section 4) could be interpreted as

251 shape variation driven by differences in floral curvature. However, the limitation of landmark- 
252 based GM in the quantification of curvature is that this method is concerned with analyzing the

253 entirety, not segments, of a specimen's shape. Outline-based GM, though well-suited to

254 analysing open curve segments (MacLeod, 1999), lacks a method for specifically extracting

255 information about curvature (detailed in Section 4). Therefore, while GM has enabled the

256 quantification of shape (sensu Bookstein, 1991), we are currently limited to describing curvature

257 by visual inspection of the principal axes of shape space. 


\section{Curvature: concepts from differential geometry}

260 Reviewing the literature leads us to ask, "what is curvature?" In related fields, for instance in

261 plant physiology, there have been uses of a point-wise definition of curvature resembling that

262 used in differential geometry (Castle, 1962). However, as in pollination ecology, references to

263 the mathematical literature are missing. Therefore, we propose turning to the field of geometry in

264 order to develop the concept of curvature starting from first principles. There, we again find

265 several definitions resulting from a history of independent derivations (reviewed in Coolidge,

266 1952; Bardini and Gianella, 2016). Nonetheless these definitions share a conceptual theme;

267 curvature is a local property that can be measured point-wise on a line. This concept is

268 fundamentally different from those typically used in pollination ecology, where curvature is a

269 single property of an entire shape. Here we follow the conventions of Casey (1996) and Rutter

270 (2000) and present a definition of curvature that is tractable for analyzing biological shapes.

271 Intuitively, when a line deviates from being straight we say it is curved, the extent to which

272 it is not straight is its curvature. More technically, a line deviates from being straight when its

273 slope (i.e. the graph of the first derivative) changes magnitude - this is represented here by the

274 rotating unit tangent vectors $\mathbf{T}_{0}, \mathbf{T}_{2}, \mathbf{T}_{7}, \mathbf{T}_{n}$ in Figure 2 . Therefore, curvature can be thought of as

275 the rate of change in the tangent as we move across the curve. Hence, the tangents of a straight

276 line will have the same slope everywhere, and the line will have a curvature of zero, whereas

277 when the slopes of the tangents of a curve (Figure 2) fluctuate, the line will have non-zero

278 curvature.

279 As biological curves often loop back on themselves (e.g. spirals) they are best described by

280 parametric functions. By using a 'hidden' variable that determines the values of $x$ and $y$ 
281 independently, parametric functions allow a curve to have multiple $y$ values for a single $x$. Here,

282 we use the parameter variable arc length, $s$, along the curve, to give us the $x$ and $y$ position.

283 Specifically, we can express a position vector $\mathbf{r}=[x, y]$ as a function solely of arc length, $s$.

284 Using vector notation we have:

$$
\mathbf{r}\left(s_{i}\right)=\mathbf{r}_{i} \equiv\left[\begin{array}{l}
x\left(s_{i}\right) \\
y\left(s_{i}\right)
\end{array}\right]
$$

286 Here $\mathbf{r}_{i}$ is shorthand for $\mathbf{r}\left(s_{i}\right)$, which indicates that our position $\left(x\left(s_{i}\right), y\left(s_{i}\right)\right)$ on the curve is

287 determined by the length of the segment $s_{i}$. Although we could parameterize a curve by many

288 potential parametric variables, arc length is a convenient choice because it allows us to move

289 along the curve at uniform increments, which we denote as $\Delta s$. This proves useful when taking

290 repeated, equally-spaced measurements (such as curvature) along a curve.

291 As we are interested in the derivative properties of our arc-length parameterized curve, we

292 can differentiate $\mathbf{r}(s)$ with respect to arc length $s$ in the following way (using the formal

293 definition of the derivative):

$$
\lim _{\Delta s \rightarrow 0} \frac{\Delta \mathbf{r}(s)}{\Delta s}=\frac{d \mathbf{r}(s)}{d s}=\mathbf{T}(s)
$$

295 This produces a tangent function $\mathbf{T}(s)=\frac{d \mathbf{r}}{d s}$ giving the first derivative of the parametric equation

$296 \mathbf{r}(s)$. The tangent $\mathbf{T}\left(s_{i}\right)$, represented by the shorthand $\mathbf{T}_{i}$, contains information about the

297 direction of the curve at position $\mathbf{r}_{i}$ that we can use to calculate curvature. When the tangent $\mathbf{T}$ is

298 placed into a cartesian plane, we can re-parameterize by the angle $\phi$ formed with the $x$-axis

299 (Figure 2). Thus the $x^{\prime}\left(s_{i}\right)$ and $y^{\prime}\left(s_{i}\right)$ components of the tangent vector $\mathbf{T}_{\mathbf{i}}$ can be expressed as:

$$
\mathbf{T}_{\mathbf{i}}=\left[\begin{array}{l}
x^{\prime}\left(s_{i}\right) \\
y^{\prime}\left(s_{i}\right)
\end{array}\right]=\left[\begin{array}{l}
\cos \left(\phi_{i}\right) \\
\sin \left(\phi_{i}\right)
\end{array}\right]
$$


301 Where:

302

$$
\tan \left(\phi_{i}\right)=\frac{y^{\prime}\left(s_{i}\right)}{x^{\prime}\left(s_{i}\right)}
$$

303 And:

$$
\phi_{i}=\arctan \frac{y^{\prime}\left(s_{i}\right)}{x^{\prime}\left(s_{i}\right)}
$$

At the beginning of this section we defined curvature, $\kappa$, as the rate at which the tangent is

306 changing direction. Thus, curvature $\kappa$ can be expressed as the change in the angle $\phi$ formed

307 between the tangent $\mathbf{T}$ and the $x$-axis:

$$
\kappa=\frac{d \phi}{d s}
$$

309 This definition provides an intuitive unit of measurement for reporting curvature: degrees of

310 rotation per unit arc length (Figure 2). For example, if curvature has been calculated at every

311 millimeter along the length of an arc, we would report its curvature as degrees per millimeter

312 degrees $\cdot \mathrm{mm}^{-1}$. Framed this way curvature is a measure of rotation per unit distance. In

313 contrast to previous definitions, where curvature is an indivisible, single property of an entire

314 shape, here, curvature is a property of every measured point along the curve. Under this point-

315 wise definition, we can summarize the total curvature (Milnor, 1954) of a specimen as the total

316 point-wise individual curvature along the curve:

$$
\kappa_{\text {total }}=\int_{0}^{s_{\max }} \kappa d s
$$

318 Units for total curvature are no longer expressed as degrees $\cdot \mathrm{mm}^{-1}$ because we are not 319 measuring curvature at a single point. Instead we are integrating all tangent rotations along the 
curve, expressed simply as degrees. For two curves of the same shape (sensu Bookstein, 1991),

321 their total curvature will be equal, regardless of allometric scaling. For example, compare the

322 half-unit circle with another half-circle with $r=2$. Although the second curve is larger, the total

323 curvature of both is $\pi$ radians. For comparative studies that need to account for size, we suggest

324 dividing total curvature by arc length, $s$, so that adjusted total curvature is:

$$
\kappa_{a d j}=\frac{\kappa_{\text {total }}}{s}
$$

Using the example above, the half-unit circle would have $\kappa_{a d j}=\frac{\pi}{\pi}=1$ radians, while the half-

327 circle with $r=2$ would have $\kappa_{a d j}=\frac{\pi}{2 \pi}=\frac{1}{2}$ radians. Shape being equal, smaller curves will

328 have greater adjusted total curvature. However, the use of this adjustment depends greatly on the

329 biological context of the research question (Klingenberg, 2016).

This concept of curvature has been embedded within the morphometrics literature since the

331 1970's. The widely-applied tangent angle function $(\phi(t))$ defined by Zahn and Roskies (1972)

332 describes complex shapes by measuring the tangent angle $(\phi)$ many times along a shape's

333 perimeter. This approach to describing shape is analagous to following cardinal directions in

334 wayfinding. Starting from the first landmark, the tangent angle $(\phi)$ between landmarks is

335 calculated step-wise around the shape. This step-wise progression eventually outlines the shape's

336 perimeter (MacLeod, 2011). The resultant tangent angle graph is a unique single-valued function

337 describing the specimen's shape and implicitly contains information about point-wise curvature.

338 However, because this technique was developed for quantifying shape, its utility as a curvature

339 metric has generally not been recognized or applied (but see: Van Otterloo, 1991). This point is

340 discussed further in the following section. 
bioRxiv preprint doi: https://doi.org/10.1101/2021.10.05.429567; this version posted October 6, 2021. The copyright holder for this preprint (which was not certified by peer review) is the author/funder, who has granted bioRxiv a license to display the preprint in perpetuity. It is made available under aCC-BY 4.0 International license.

341 


\section{A proposed protocol for measuring curvature}

343 As illustrated in our methodology review, the current protocols for measuring flower-pollinator

344 curvature lack conceptual unity. There are two main advantages of the curvature definition

345 described above. First, curvature becomes a local property of the tissue or organ under study.

346 This means that shape information is gathered at every point along the curve and can be

347 examined and compared to other points within or between specimens. This differs from previous

348 methods that take curvature as an indivisible property of the entire specimen. Second, because

349 the revised definition comes directly from the field of differential geometry, we benefit from

350 established, well-defined concepts that make clear what is meant by 'curvature.' When the

351 definition of curvature is concordant between these research areas, future advances in geometry

352 can be more readily incorporated into morphological studies.

353 In order to apply the point-wise definition of curvature, a biological organ or tissue needs

354 to be reduced to a continuous function. To do this, we propose a protocol as illustrated in Figure

355 3. First, a specimen is landmarked at several locations along the region of study. Second, a

356 mathematical function is fitted to the landmarks, and finally, curvature is calculated point-wise

357 along the curve. The long-term goal of this proposal is to integrate the analysis of curvature with

358 existing morphometrics protocols. In the following demonstration we use existing morphometric

359 tools for landmarking and curve-fitting - these were previously developed in the field of

360 traditional and outline morphometrics (e.g. Rohlf, 1990; MacLeod and Rose, 1993; Terral et al.,

3612004 , Section 2).

362 As mentioned in the previous section, the field of plant physiology was an early adopter of

363 the point-wise definition of curvature. In one case we found curvature (as defined above) 
364 computed from cubic functions fitted to cucumber seedlings that had been landmarked by hand

365 (Cosgrove, 1990). Our protocol can be seen as a computerized version of this procedure. In

366 another study, total curvature of Anthurium Schott (Araceae) spadices was computed from fitted

367 B-spline curves (Pour et al., 2018). However, because landmarks were not defined and the scripts

368 are not publicly available, the reproducibility of this protocol is low. Here, we propose to

369 develop the analysis of curvature specifically within the R programming environment (R Core

370 Team, 2017), where existing landmarking and curve-fitting procedures can be called, and where

371 modern morphometrics is being most actively developed (e.g. Adams and Otárola-Castillo, 2013;

372 Bonhomme et al., 2014).

In the following section we calculate curvature from cubic smoothing splines (Wood, 2017)

374 fitted to landmarks. While splines are suitable for relatively simple, open curves, we encourage

375 the development of algorithms that will compute curvature from more sophisticated curve-fitting

376 strategies (reviewed in Rohlf, 1990; MacLeod, 2002). Already there is potential for existing GM

377 algorithms to be modified to compute curvature. Notably, the tangent angle function, $\phi(t)$,

378 describes the angle of the tangent at a landmark as a function of distance traveled along the

379 specimen's outline (Zahn and Roskies, 1972; MacLeod, 2012). While the concept of $\phi$ (sensu

380 Zahn and Roskies, 1972) is equivalent to the one described in Section 3, the tangent angle

381 function appears to have been derived without reference to differential geometry (Raudseps,

382 1965). Though we are interested specifically in the curvature of shapes, the tangent angle

383 function was developed as a means to quantify shapes in their entirety-curvature is incidental.

384 Nonetheless, when many interpolated semi-landmarks are used to model a specimen's outline

385 (e.g. Zahn and Roskies, 1972), the tangent angles could be summed to give an approximation of 
bioRxiv preprint doi: https://doi.org/10.1101/2021.10.05.429567; this version posted October 6, 2021. The copyright holder for this preprint

(which was not certified by peer review) is the author/funder, who has granted bioRxiv a license to display the preprint in perpetuity. It is made available under aCC-BY 4.0 International license.

$386 \kappa_{\text {total }}$. There is great opportunity for existing tangent angle algorithms (e.g. Claude, 2008) to

387 parse curvature data as a part of an outline morphometrics analysis. 


\title{
Demonstration: A study of the development of curvature in Epimedium
}

\author{
Study System and Methods
}

In this demonstration, we compare the development of floral morphology in two subspecies from the Epimedium grandiflorum complex (Stearn, 2002): Epimedium koreanum Nakai related, these taxa have notable differences in floral pigmentation and size (Stearn, 2002). We investigated whether there were consequent differences in floral shape, including curvature of

397 the prominent nectar spurs. This study serves to demonstrate that the analysis of curvature can be 398 improved by clearly defined protocols and units of measurement, in addition to encouraging the integration of traditional and geometric morphometrics. nectar spurs ( $n=57$, Table S2) of varying maturity were sampled for imaging. Spurs were

402 photographed in the dorsiventral view using a stereo microscope at 6.3x. Flower shape was 403 quantified from the images using a standard GM protocol in geomorph v.3.2.0 (Adams and 404 Otárola-Castillo, 2013), as outlined in Section 4 (see also: Supp. Methods). Shape data was then 405 analysed for the principal components (PCs) describing the major axes of variation in shape 406 space (Figure 4).

Morphological development can be compared by quantifying the size, orientation, and

408 shape of an individual's trajectory through morphometric shape space (Adams and Collyer, 409 2009). We used developmental trajectory analysis (Collyer et al., 2015) to determine how shape 410 varied with growth stage. First, a linear mixed model was fit to relate changes in shape with 
411 developmental stage (model variables and likelihood ratio tests for significance are described in

412 the Supp. Materials):

$$
\text { shape }=\beta_{0}+\beta_{1} * \text { stage } * \text { taxon }+\beta_{2} * \text { individual }+\epsilon
$$

414 This model was submitted to geomorph: :trajectory.analysis() to quantify and compare

415 developmental trajectories between the two taxa. Pairwise distances of shape means for each

416 stage were calculated in RRPP v.0.5.2 (Collyer and Adams, 2018).

417 The deformation grids illustrating variation in PCA shape space suggested that floral

418 curvature increased along both PC1 and PC2 (Figure 4). To analyse curvature specifically,

419 smoothing splines (Cleveland et al., 1992) were fitted to the dorsal side of the nectar spurs using

420 stats: : smooth.spline() (Supp. Methods). Total curvature (Section 3) was calculated from

421 the splines using the R package curvr v.0.1.0 (Boehm, 2020). To test for the correlation between

422 the PCs of shape space and total curvature we fit the linear mixed model:

$$
P C 1=\beta_{0}+\beta_{1} * \kappa_{\text {total }} * \text { taxon }+\beta_{2} * \text { individual }+\epsilon
$$

425 varied with age (Supp. Methods). Estimated marginal means and pairwise comparisons were 426 calculated for each taxon and developmental stage using the emmeans package v.1.4.5 (Lenth et 427 al., 2018).

428 Finally, we re-measured curvature using metrics 2 to 5 as outlined in our summary of the 429 previous literature (Section 2, Supp. Methods), and performed a pairwise regression for each 430 metric, including the point-wise defintion proposed here. Linear measurements (e.g. chord 431 length) were made using tpsDig v.2.3.2 (Rohlf, 2015). The inverse radius was computed from 432 fitted circles using pracma v.2.2.9 (Borchers, 2019). In order to estimate deviations from 
433 circularity, the root mean square error was computed for each specimen fitted by the inverse

434 radius method.

\section{Results}

Inspection of the deformation grids associated with PC1 and PC2 suggests that curvature is

438 associated with both axes of shape space (Figure 4). Indeed, there is a significant correlation

439 between curvature and PC1 $(p=0.019, t-$ ratio $=2.40)$ and PC2 $\left(p=2.1 \times 10^{-11}, t-\right.$

440 ratio $=8.49$, Figure 5). There are no interspecific differences in curvature during floral

441 development, but in E. koreanum there is a significant increase in curvature during Stage 2

442 (Table S6, Figure S6). From mid to late development (Stages 2 to 4) there is 50.6 degrees less

443 total curvature in the nectar spurs of E. koreanum ( $p=0.002$, Table S6). Similarly, from early

444 to mid development (Stages 1 to 2 ) there is 37.9 degrees less total curvature $(p=0.073)$. The

445 specimen photographs (Supp. Materials) suggest that at Stage 2, nectar spurs tend to curve

446 sharply as they elongate (grow) from bud-size spurs (Stage 1) to mature-size. After growth

447 slows, the spurs straighten as they approach anthesis (Stage 4). Whether this developmental

448 pattern is adaptive or incidental is yet unknown.

Trajectory analysis found that interspecific and temporal differences in shape are driven

450 mainly by PC1, which accounts for $61.4 \%$ of shape variation (Table S5, Figure 4). Although

451 both PCs are correlated with curvature, E. koreanum at Stage 2 did not necessarily deviate from

452 other samples in morphospace (Table S5). In this case, it may be that other geometric

453 components contribute to spur shape, and curvature alone does not sufficiently explain variation

454 along the PCs of shape space. 
Pairwise comparisons of the various curvature metrics found that, for both species, all

metrics are significantly correlated ( $p<0.0043$, Tables S8 and S9). The inverse radius had the

457 lowest correlation with the other metrics (Pearson's $r<0.65$ ). Thus, we used

458 pracma: : circlefit() to calculate the error distance for each specimen's fitted circle.

459 Consequently, the specimens approximated poorly by circles had the most residual variation

460 between total curvature and the inverse radius metric $\left(p=3.78 \times 10^{-7}, t=5.7\right.$, Figure S8).

461 This suggests that for non-circular curves, a point-wise metric is better suited to capture local

462 deviations and fluctuations in curvature.

\section{Discussion and Future Applications}

The proposed definition for curvature (as adopted from differential geometry) clarifies both

466 the concept and the units of measurement. Using the terms point-wise curvature and total

467 curvature enables us to distinguish between the curvature at a point and the cumulative curvature

468 of the structure under study. In the context of pollination ecology, a rigorous measure of

469 curvature will facilitate a number of avenues of investigation. These include matching curvature

470 of flowers and bills in co-evolutionary studies, describing the variation of curvature of flowers

471 and bird bills in populations as raw material for evolution, and investigating the evolution of

472 curved flowers and bird bills in the context of phylogeny.

473 By pairing an analysis of curvature with geometric morphometrics, we are able to quantify

474 the covariation between curvature and overall shape. Further integration of univariate metrics

475 with GM could be achieved by modifying the principal component analysis of shape to partial

476 out variation due to an explanatory variable (e.g. redundancy analysis, Borcard et al., 2018). 
477 Because GM is ubiquitous among studies of biological form, we hope that this metric for 478 curvature, as presented here, may facilitate communication via a common language between

479 fields that are interested in curved structures. The diversity of applications spans the study of

480 sexual selection on horn, tusk, and antler structure (e.g. in dung beetles Emlen et al., 2005), the

481 twining and nutation of tendrils (Goriely and Neukirch, 2006; Bastien et al., 2014), the functional

482 and behavioural ecology of claw shape (e.g. in birds, lizards, and dinosaurs Birn-Jeffery et al.,

483 2012), the bending of hypocotyls in response to gravity and light (Silk, 1989; Frassen et al.,

484 1982), and the biomechanics of locomotion and escape behaviours (e.g. prehensile tails in

485 Neotropic monkeys, Schmitt et al., 2005; lateral bending of lizards, Ritter, 1992). Widening the

486 applicability of this curvature metric will require drawing deeper from the morphometrics

487 toolkit. Beyond simple curves that might be fitted by polynomials, splines are necessary for 488 describing spirals, undulating lines, and multi-valued functions. For closed outlines, ZR Fourier 489 Analysis (Section 2, Zahn and Roskies, 1972; MacLeod, 2011) is well-suited for decomposing a 490 range of complex biological shapes. As discussed in Section 3, the tangent angle function used to

491 represent a closed outline mirrors the defintion of curvature used in differential geometry.

492 Fortunately, Momocs (Bonhomme et al., 2014) offers functions for ZR Fourier Analysis,

493 providing a convienient launch point for integrating point-wise curvature estimation with the

494 quantification of closed outlines. With much of the groundwork already laid, estimating point-

495 wise curvature could soon be applied to a diversity of study systems. 
500 In this synthesis we discussed the ecological significance of curvature within the field of

501 pollination ecology. In reviewing the methods used to measure curvature, we found a need for

502 both conceptual and methodological unity. By drawing from the geometry literature we aimed to

503 clarify the definition of curvature within the contexts most commonly used in pollination

504 ecology: the curvature of floral tubes or pollinator mouthparts in the dorsiventral plane. We

505 demonstrated the utility of this revised metric by analysing the development of curved nectar

506 spurs in Epimedium. For curves that deviate from segments of circles, a point-wise definition of

507 curvature is recommended over historical methods that model circularity on segments of a circle.

508 Furthermore, a clearly defined concept of curvature creates the potential for progressive

509 integratation with the swiftly advancing, and broadly applied field of geometric morphometrics. 

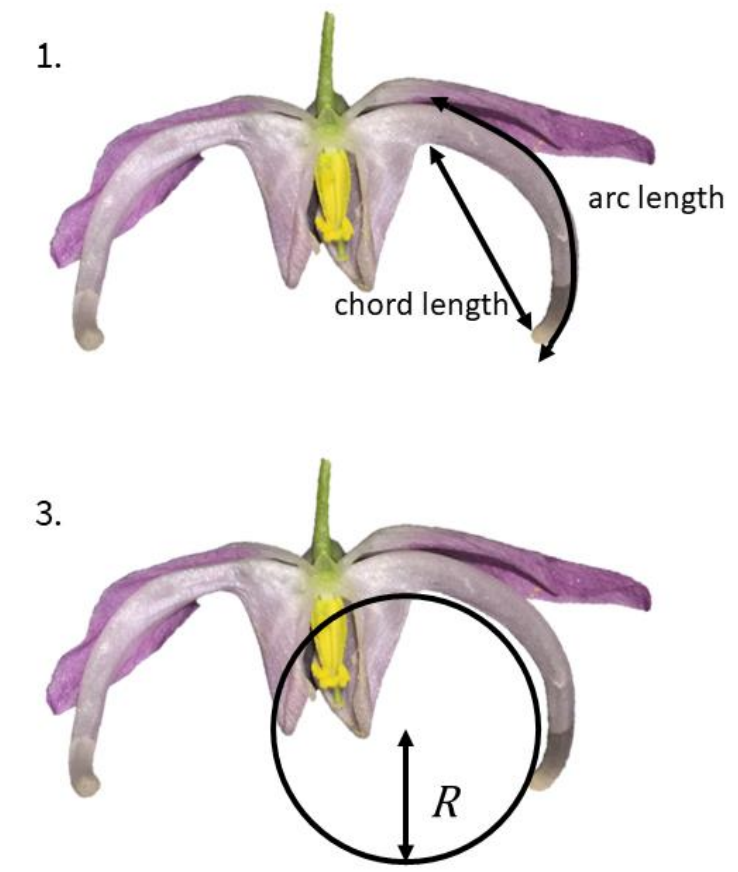

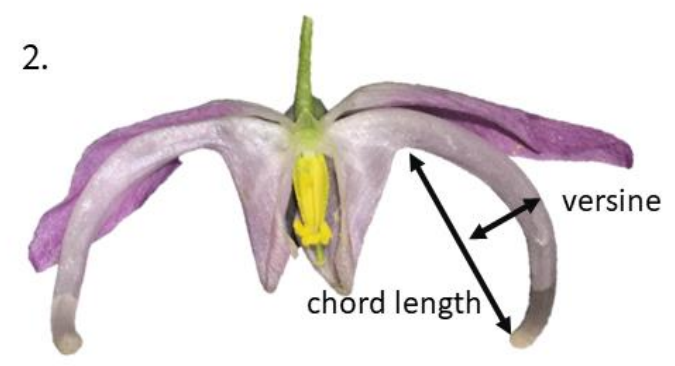

4.

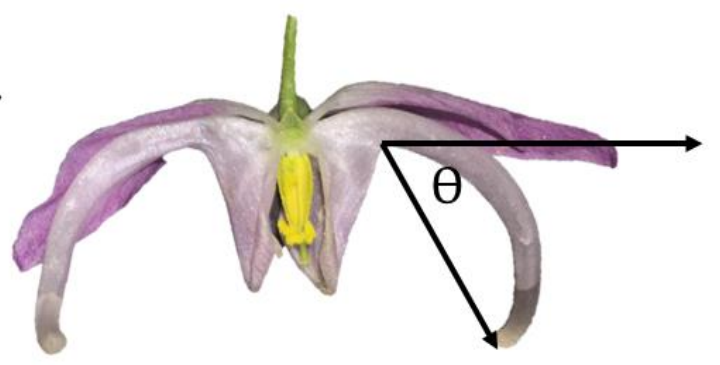

512 Figure 1. Overview of most commonly used curvature metrics within pollination ecology. 1.

513 arc:chord ratio. 2. mandibular index 3. inverse radius. 4. angle of deflection 


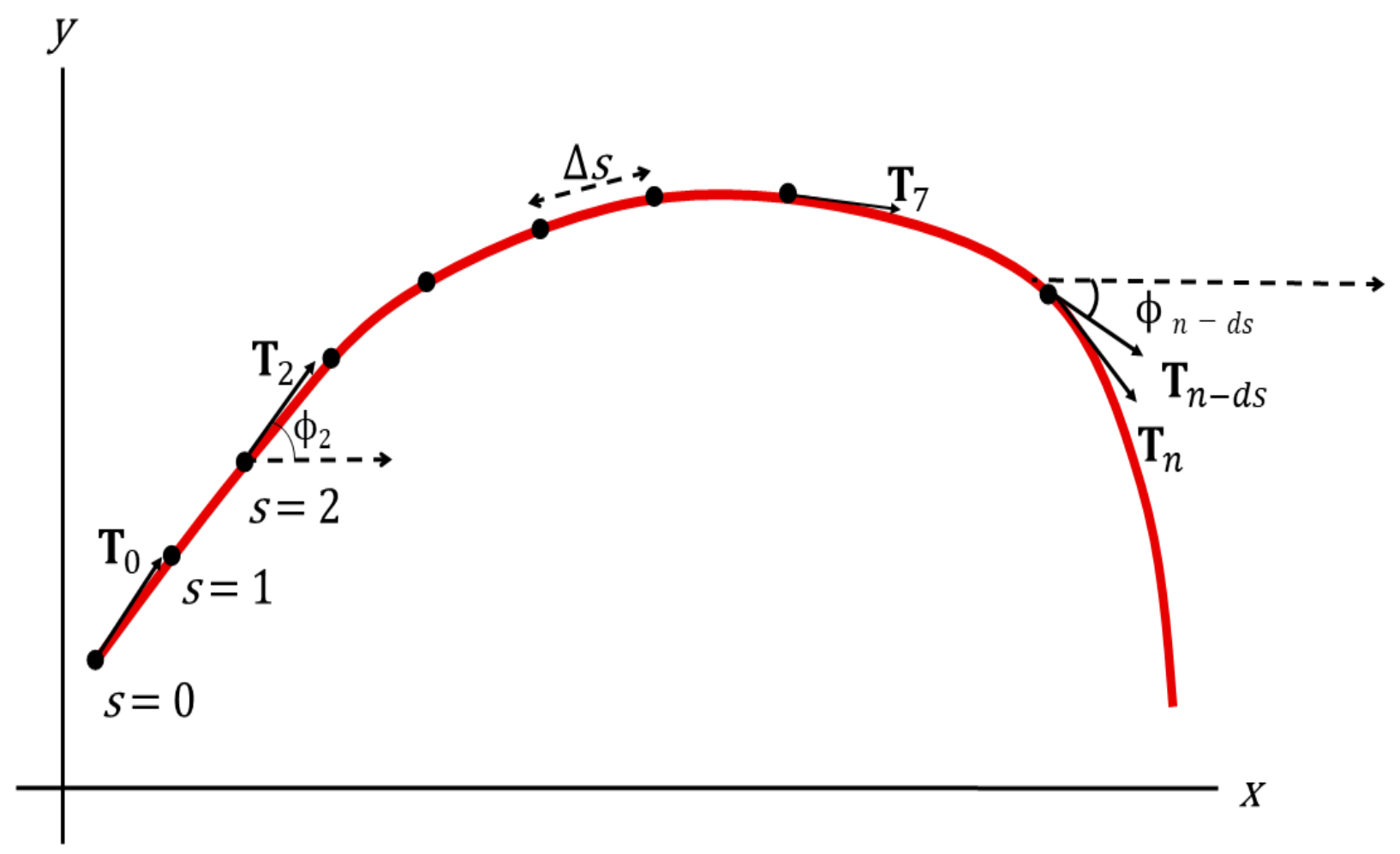

517 Figure 2. A curve parameterized by arc length, s. $T_{0}, T_{2}$, and $T_{7}$ are the tangents $\left(\frac{d \boldsymbol{r}}{d s}\right)$ at $s=0$,

$518 s=2$, and $s=7$, respectively. For convenience the tangents are displayed as indications only,

519 not exact representations. $\phi_{2}$ and $\phi_{n-d s}$ represent the angles formed between the tangent $T_{2}$ and

520 the $x$ axis, and $T_{n-d s}$ and the $x$ axis, respectively. Total curvature is the sum of the changes in

521 rotation $(\Delta \phi)$ along the curve. 

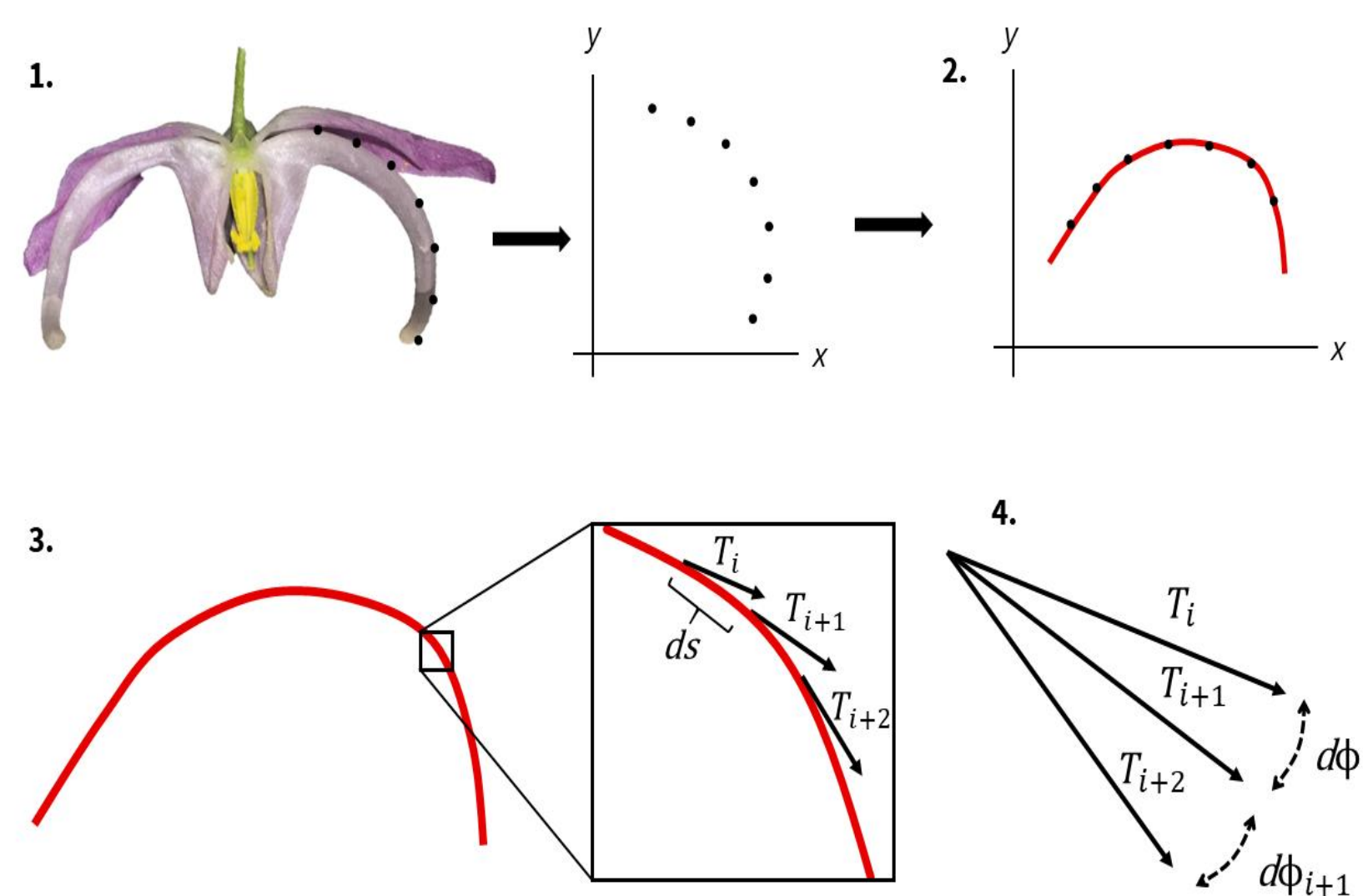

4.

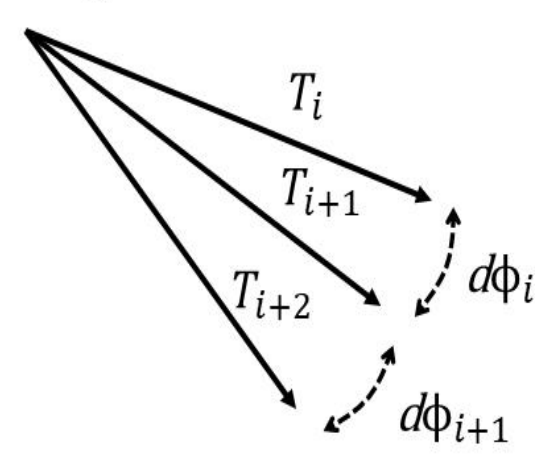

524 Figure 3: Proposed protocol for measuring curvature. 1. A specimen (in this case Epimedium

525 violaceum) is landmarked, semi-landmarked and assigned xy coordinates within a Cartesian

526 plane. 2. The xy coordinates are rotated so that a single-valued function can be fitted to the

527 landmarks (this step is not necessary for all cases). 3. The tangent vector $\boldsymbol{T}$ is calculated at an

528 arbitrarily large number of increments, $d s$, along the curve. 4. Curvature is calculated as the

529 rate of change of the tangent angle ( $\phi$ ) point-wise along the curve. Total curvature is calculated

530 by the methods outlined in Section 3. 

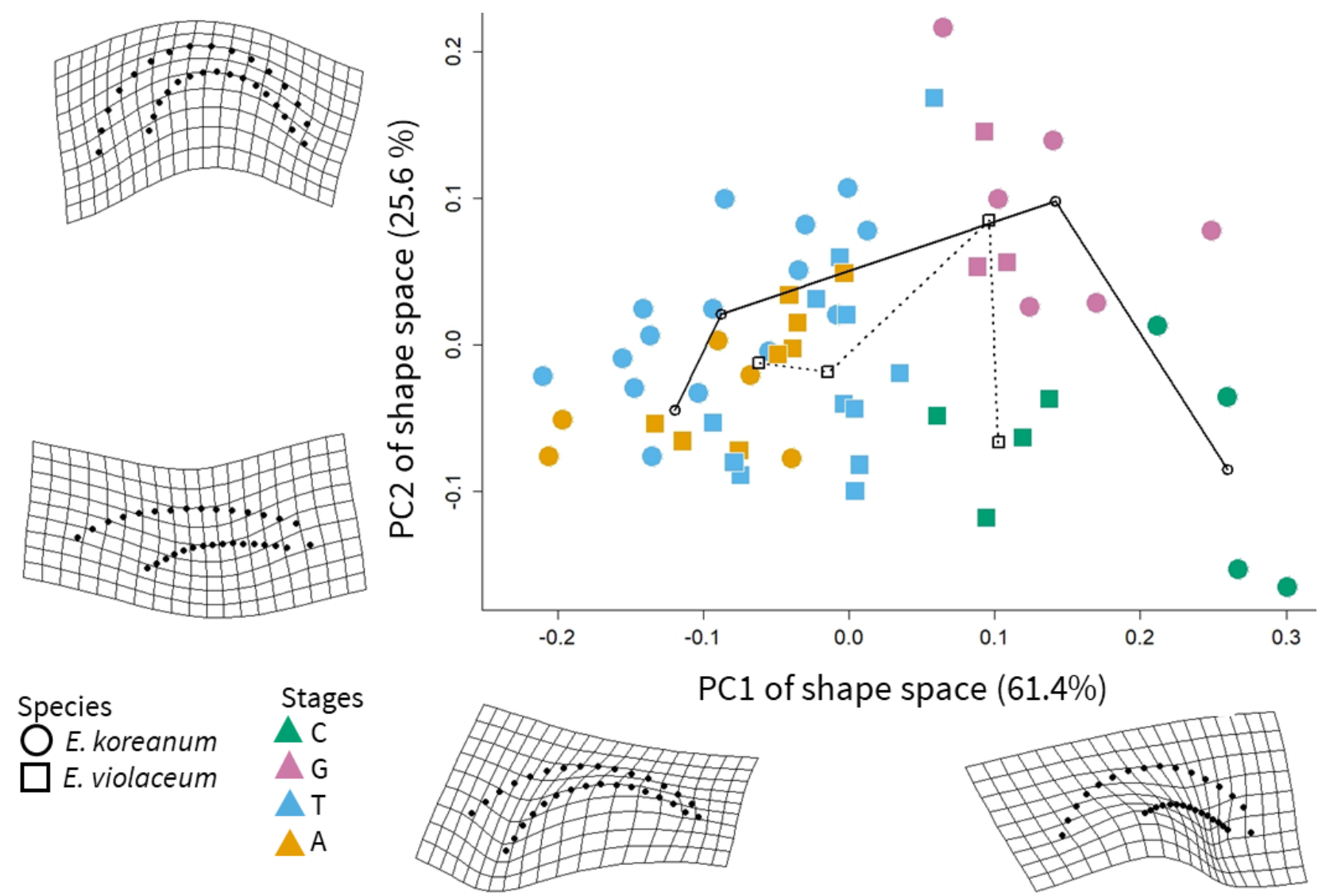

533 Figure 4: Morphometric trajectory analysis of nectar spur development in E. koreanum (circles)

534 and E. violaceum (squares). The PC axes and deformation grids illustrate the two most

535 explanatory components of shape change in the development of nectar spurs. The two taxa

536 studied occupy significantly different domains of morphospace at the first (green) stage of

537 development. At maturity (yellow), the two species do not differ significantly in shape. 

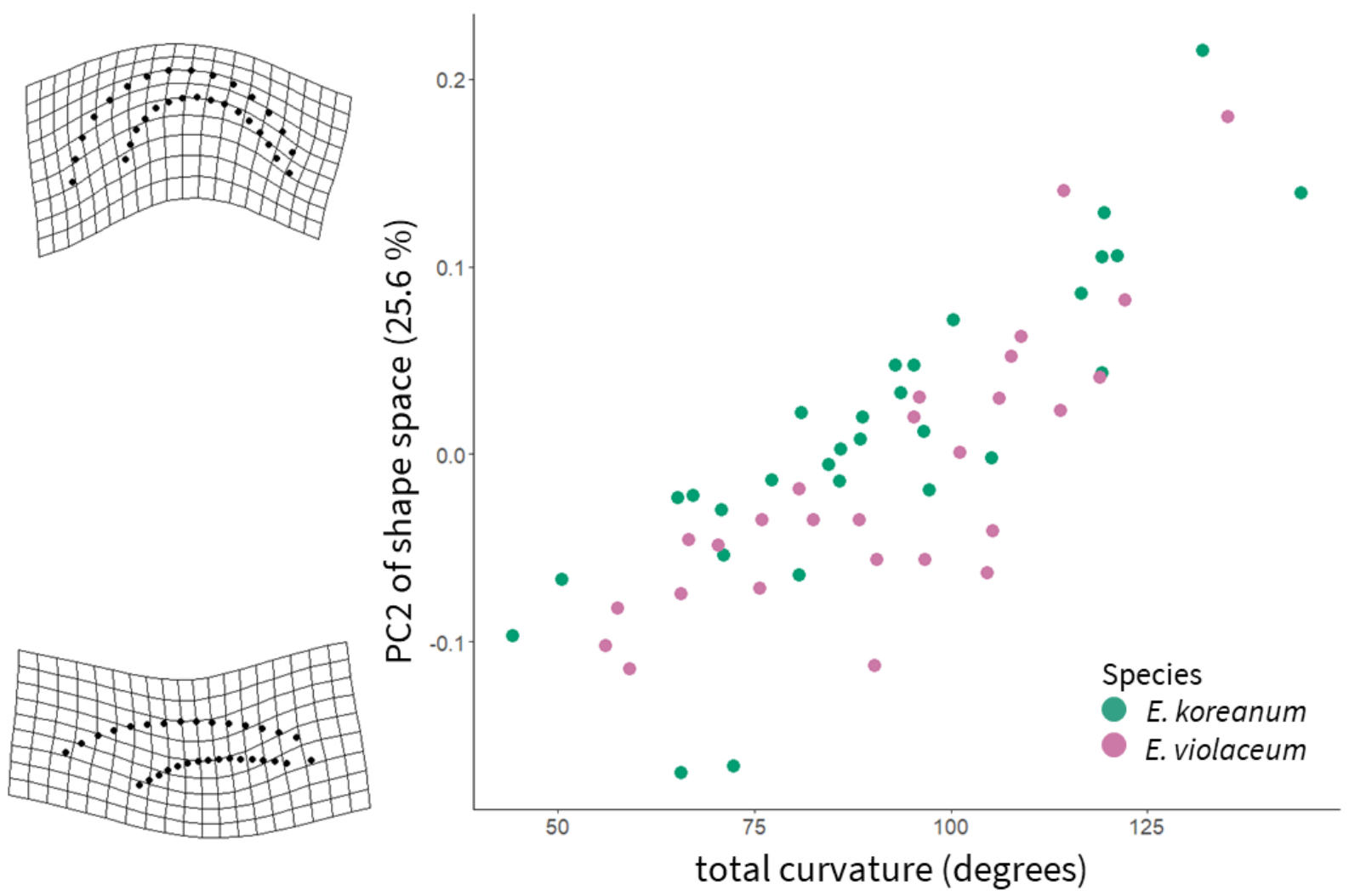

540 Figure 5: Scatterplot of total curvature and PC2 from PCA of geometric morphometric shape

541 data (see also: Figure 4). PC2 represents the axis of second greatest variation in shape during

542 Epimedium flower development. 
544 Table 1: Summary of literature reviewed for metrics of floral or mouthpart curvature in plant-

545 pollinator systems.

Stated or Inferred

Citation

System

Method

Snow and Snow

Bill morphology and niche partitioning of nine

qualitative

hummingbird species in the Arima Valley,

Trinidad.

Stiles (1975)

Corolla morphology of Heliconia (Zingerberales) qualitative

and bill morphology of nine hummingbird species

at La Selva, Costa Rica.

Feinsinger and

Bill morphology and niche partitioning within

qualitative

Colwell (1978)

hummingbird communities of the Carribean

Islands and Monteverde, Costa Rica.

Gill and Wolf

Sunbird bill diversity and abilities to extract

mandibular index

nectar from Kenyan Leonotis nepetifolia

(Lamiaceae).

Carothers (1982) Variation in bill morphology in three species of angle of deflection

Hawaiian honeycreeper, and effects on feeding

performance from Vaccinium calycinum

(Ericaceae). 
Grant and Grant

Mountainspring

Paton and Collins

Muller (1995)

Stiles (1995)
Effects of Hawkmoth proboscis length on pollination of Mirabilis longiflora

(Nyctaginaceae).

Sexual dimorphism and foraging preferences of the Maui Parrotbill (Pseudonestor xanthophrys).

Functional ecology of bill shape in hummingbirds, honeyeaters, sunbirds, and

Hawaiian honeycreepers.

Curved bristles on the proboscis of European bees for the extraction of pollen.

Smith et al. (1995)

Correlated evolution of diet and bill shape in

Hawaiian honeycreepers.

Effects of bill morphology on insect foraging strategy by 11 species of hummingbirds at La

Selva, Costa Rica.

Mclntyre and

Browne (1996)

Manning and

Goldblatt (1997)
Phototropism in Helianthus (Asteraceae) and effects on cotyledon curvature.
Iridaceae, Geraniaceae, and Orchidaceae of South qualitative

mandibular index

mandibular index inverse radius

arc:chord ratio angle of deflection

qualitative

Africa. 
Cotton (1998)

Temeles et al.

Borgella Jr et al.

Temeles and Kress

Travers et al. (2003)

Temeles et al.
Survey and description of 16 hummingbird

species occurring at Amacayacu National Park,

Colombia.

Sexual dimorphism of bill shape in Purple-

throated caribs (Eulampis jugularis) and effects

on pollination of Heliconia at Quilesse Reserve,

Saint Lucia.

Effects of bill morphology (21 hummingbird

spp.) on pollen loads (35 plant spp.) at Coto Brus,

Costa Rica.

Sexual dimorphism of bill shape in Purple-

throated caribs (Eulampis jugularis) and effects

on pollination of Heliconia at Saint Lucia and

Dominica.

Nectar spurs of Impatiens spp. and Ruby-throated hummingbird (Archilochus colubris) at Franklin

County, Massachusetts.

Sexual dimorphism of bill shape in Purple-

throated caribs (Eulampis jugularis) and effects

on pollination of Heliconia at Quilesse Reserve,

Saint Lucia. angle of deflection

qualitative

angle of deflection, inverse radius

arc:chord ratio

angle of deflection

angle of deflection, inverse radius 
Collins (2008)

Stiles (2008)

Young (2008)

Martén-Rodríguez et

al. (2009)

Temeles et al.

Luo and Li (2010)

Temeles et al.

Berns and Adams

(2010)
Foraging efficiency from artificial and natural (15 mandibular index

spp.) flowers by 4 species of hummingbirds at

Monteverde, Costa Rica.

Correlations of bill morphology to the elevational arc:chord ratio

distributions of $150 \mathrm{spp}$. of hummingbirds in the

Andes.

Effects of spur shape on male and female fitness

angle of deflection

in Impatiens capensis (Balsaminaceae).

angle of deflection Antillean Gesneriaceae.

Effects of natural (Heliconia) and artificial flower angle of deflection, morphologies on foraging performance of Purple- inverse radius throated caribs (Eulampis jugularis) at Saint

Lucia.

Effects of light and temperature on style

angle of deflection curvature in Alpinia (Zingiberaceae).

Sexual dimorphism of bill shape in 21 species of

angle of deflection,

Central and South American hummingbirds.

inverse radius

Sexual dimorphism of bill shape in black-chinned geometric

hummingbird (Archilochus alexandri) and ruby- morphometrics

throated hummingbird (Archilochus colubris). 
Berns and Adams $\quad$ Sexual dimorphism of bill shape in 219

(2013)

Wang et al. (2013)

hummingbird spp.
Selection for nectar spur curvature in Impatiens

oxyanthera (Balsaminaceae) mediated by

pollinators and nectar robbers.

Maglianesi et al.

Rico-Guevara and

Araya-Salas (2014)

Alexandre et al.

Elevational variation in plant-hummingbird network structure mediated by bill morphology.

La Selva, Costa Rica.

Selection for bill shape driven by male-male

competition in Phaethornis longirostris. La

Selva, Costa Rica.

QTL analysis comparing hummingbird pollinated and generalist Rhytidophyllum flowers

(Gesneriaceae).

Campos et al. (2015) Generating 3D printed flowers to experimentally study the feeding mechanics of moth pollination.

Maglianesi et al. (2015b)
Differential preferences of artificial and natural (65 spp.) flower populations visited by 3 species

of hummingbird in Braulio Carrillo National Park, Costa Rica. geometric

morphometrics

angle of deflection

angle of deflection

arc:chord ratio

angle of deflection

curve decay

parameter

angle of deflection 
Maglianesi et al. Plant-pollinator specialization along an (2015a)

Rocha et al. (2015)

Miller et al. (2017)

Lagomarsino et al. (2017)

Boehm (2018)

Joly et al. (2018)

plant species examined.

Physical and biochemical basis for

Passiflora mucronata (Passifloraceae).

Campanulaceae.

(Campanulaceae)

specialization. Coto Brus, Costa Rica.
Hadley et al. (2018)

elevational gradient at Braulio Carrillo National

Park, Costa Rica. 21 hummingbird spp. and 208

androgynophore bending in bat-pollinated

Ecological divergence among closely related,

morphologically similar Honeyeaters co-

occurring in arid Australian environments.

Evolution of pollination syndromes in Andean

Review of nectar robbing in Centropogon

Effects of forest fragmentation on hummingbird bill morphologies (19 spp.) representative of

Testing the pollination syndrome hypothesis in

Antillean Gesneriaceae. arc:chord ratio

angle of deflection

arc:chord ratio

arc:chord ratio

arc:chord ratio

qualitative

geometric

morphometrics 
Partida-Lara et al. Spatio-temporal structure of the taxonomic and inverse radius

functional diversity of hummingbirds at the

biosphere reserve El Triunfo, Chiapas, Mexico

Dellinger et al. $\quad$ Floral trait changes correlated with the repeated

qualitative

shifts away from buzz pollination in the

Melastomataceae.

Peng et al. (2019) Evaluation of fitness optima of the moth

curve decay

proboscis and flower shape.

parameter

Sonne et al. (2019)

Variation strength of plant-hummingbird

arc:chord ratio

specialization along an elevational gradient in

Podocarpus National Park, Ecuador.

Johnson et al. (2020) Experimental effects of floral orientation and

angle of deflection curvature on nectar extraction by Amethyst sunbirds (Chalcomitra amethystina). 


\section{References}

548 Adams, D.C., and Collyer, M. (2009). A general framework for the analysis of phenotypic trajectories in evolutionary studies. Evolution 63, 1143-1154.

550 Adams, D.C., and Otárola-Castillo, E. (2013). Geomorph: An R package for the collection and analysis of geometric morphometric shape data. Methods in Ecology and Evolution 4, 393-399.

Alexandre, H., Vrignaud, J., Mangin, B., and Joly, S. (2015). Genetic architecture of pollination syndrome transition between hummingbird-specialist and generalist species in the genus

Armbruster, W.S., and Muchhala, N. (2009). Associations between floral specialization and species diversity: Cause, effect, or correlation? Evolutionary Ecology 23, 159-179.

Baldwin, S.P., Oberholser, H.C., and Worley, L.G. (1931). Measurements of birds (Cleveland Museum of Natural History).

Bardini, G., and Gianella, G.M. (2016). A historical walk along the idea of curvature, from Newton to Gauss passing from Euler. International Mathematical Forum 11, 259-278.

Bastien, R., Douady, S., and Moulia, B. (2014). A unifying modeling of plant shoot gravitropism with an explicit account of the effects of growth. Frontiers in Plant Science 5, 136-145.

564 Bell, J. (1956). Tangent, chord theorem. The Mathematical Gazette 40, 211-212.

565 Berns, C.M., and Adams, D.C. (2010). Bill shape and sexual shape dimorphism between two 635. 
Berns, C.M., and Adams, D.C. (2013). Becoming different but staying alike: Patterns of sexual size and shape dimorphism in bills of hummingbirds. Evolutionary Biology 40, 246-260.

571 Birn-Jeffery, A.V., Miller, C.E., Naish, D., Rayfield, E.J., and Hone, D.W. (2012). Pedal claw curvature in birds, lizards and Mesozoic dinosaurs-complicated categories and

Blüthgen, N., Menzel, F., and Blüthgen, N. (2006). Measuring specialization in species interaction networks. BMC Ecology 6, 9-21.

Boehm, M.M.A. (2018). Biting the hand that feeds you: Wedge-billed hummingbird is a nectar robber of a sicklebill-adapted andean bellflower. Acta Amazonica 48, 146-150.

Boehm, M.M.A. (2020). Curvr: Calculate Point-Wise Curvature From Landmarked Specimens.

579 Bonhomme, V., Picq, S., Gaucherel, C., and Claude, J. (2014). Momocs: Outline analysis using R. Journal of Statistical Software 56, 1-24.

581 Bookstein, F.L. (1991). Morphometric tools for landmark data: geometry and biology (Cambridge University Press).

Borcard, D., Gillet, F., and Legendre, P. (2018). Numerical Ecology with R (New York, USA: Springer).

Borchers, H.W. (2019). Pracma: Practical numerical math functions. R package version 2.2.5.

586 Borgella Jr, R., Snow, A.A., and Gavin, T.A. (2001). Species richness and pollen loads of hummingbirds using forest fragments in southern Costa Rica. Biotropica 33, 90-109. are highly controlled by nondietary factors. Proceedings of the National Academy of Sciences 113, 5352-5357. 
591 Campos, E.O., Bradshaw, H.D., and Daniel, T.L. (2015). Shape matters: Corolla curvature improves nectar discovery in the hawkmoth Manduca sexta. Functional Ecology 29, 462468.

594 Cardinal, S., and Danforth, B.N. (2013). Bees diversified in the age of eudicots. Proceedings of the Royal Society B: Biological Sciences 280, 20122686.

Carothers, J.H. (1982). Effects of trophic morphology and behavior on foraging rates of three Hawaiian honeycreepers. Oecologia 55, 157-159.

Casey, J. (1996). Exploring Curvature (Braunschweig, Germany: Friedr. Vieweg \& Sohn Verlagsgesellschaft $\mathrm{mbH})$.

600 Castle, E.S. (1962). Phototropic curvature in Phycomyces. The Journal of General Physiology 45, $743-756$.

602 Claude, J. (2008). Morphometrics with R (New York, USA: Springer Science).

603 Cleveland, W.S., Grosse, E., Shyu, W.M., Chambers, J.M., and Hastie, T.J. (1992). Statistical models in s. Local Regression Models Chapter-8.

605 Collins, B.G. (2008). Nectar intake and foraging efficiency: Responses of honeyeaters and 606 hummingbirds to variations in floral environments. The Auk 125, 574-587.

607 Collyer, M.L., and Adams, D.C. (2018). RRPP: An R package for fitting linear models to high608 dimensional data using residual randomization. Methods in Ecology and Evolution 9, $609 \quad 1772-1779$.

610 Collyer, M., Sekora, D., and Adams, D.C. (2015). A method for analysis of phenotypic change for phenotypes described by high-dimensional data. Heredity 115, 357-365.

612 Coolidge, J.L. (1952). The unsatisfactory story of curvature. The American Mathematical $613 \quad$ Monthly 59, 375-379. 
614 Cosgrove, D.J. (1990). Rapid, bilateral changes in growth rate and curvature during gravitropism 615 of cucumber hypocotyls: Implications for mechanism of growth control. Plant, Cell \& $616 \quad$ Environment 13,227-234.

617 Cotton, P.A. (1998). Temporal partitioning of a floral resource by territorial hummingbirds. Ibis $140,647-653$.

619 Dakin, R., Segre, P.S., Straw, A.D., and Altshuler, D.L. (2018). Morphology, muscle capacity, skill, and maneuvering ability in hummingbirds. Science 359, 653-657.

621 Dehling, D.M., Töpfer, T., Schaefer, H.M., Jordano, P., Böhning-Gaese, K., and Schleuning, M. (2014). Functional relationships beyond species richness patterns: Trait matching in plant-bird mutualisms across scales. Global Ecology and Biogeography 23, 1085-1093.

624 Dellinger, A.S., Chartier, M., Fernández-Fernández, D., Penneys, D.S., Alvear, M., Almeda, F., Michelangeli, F.A., Staedler, Y., Armbruster, W.S., and Schönenberger, J. (2019). Beyond buzz-pollination-departures from an adaptive plateau lead to new pollination

628 Emlen, D.J., Marangelo, J., Ball, B., and Cunningham, C.W. (2005). Diversity in the weapons of sexual selection: Horn evolution in the beetle genus Onthophagus. Evolution 59, 10601084.

631 Feinsinger, P., and Colwell, R.K. (1978). Community organization among neotropical nectarfeeding birds. American Zoologist 18, 779-795.

633 Frassen, J.M., Firn, R.D., and Digby, J. (1982). The role of the apex in the phototropic curvature of Avena coleoptiles: Positive curvature under conditions of continuous illumination. Planta 155, 281-286. 
636 Gill, F.B., and Wolf, L.L. (1978). Comparative foraging efficiencies of some montane sunbirds in Kenya. The Condor 80, 391-400.

638 Goriely, A., and Neukirch, S. (2006). Mechanics of climbing and attachment in twining plants. Physical Review Letters 97, 184302.

640 Gómez, J., Perfectti, F., Bosch, J., and Camacho, J. (2009). A geographic selection mosaic in a generalized plant-pollinator-herbivore system. Ecological Monographs 79, 245-263.

642 Graham, C.H., Parra, J.L., Rahbek, C., and McGuire, J.A. (2009). Phylogenetic structure in tropical hummingbird communities. Proceedings of the National Academy of Sciences

645 Grant, V. (1949). Pollination systems as isolating mechanisms in angiosperms. Evolution 3, 8297.

647 Grant, V., and Grant, K.A. (1983). Hawkmoth pollination of Mirabilis longiflora (Nyctaginaceae). Proceedings of the National Academy of Sciences 80, 1298-1299.

649 Hadley, A.S., Frey, S.J., Robinson, W.D., and Betts, M.G. (2018). Forest fragmentation and loss reduce richness, availability, and specialization in tropical hummingbird communities.

652 Hainsworth, F.R. (1973). On the tongue of a hummingbird: Its role in the rate and energetics of 653 feeding. Comparative Biochemistry and Physiology Part A: Physiology 46, 65-78.

654 Hughes, M., Möller, M., Edwards, T.J., Bellstedt, D.U., and De Villiers, M. (2007). The impact 
Johnson, S.D., Kiepiel, I., and Robertson, A.W. (2020). Functional consequences of flower curvature, orientation and perch position for nectar feeding by sunbirds. Biological Journal of the Linnean Society 131, 822-834.

660 Joly, S., Lambert, F., Alexandre, H., Clavel, J., Léveillé-Bourret, É., and Clark, J.L. (2018). Greater pollination generalization is not associated with reduced constraints on corolla shape in Antillean plants. Evolution 72, 244-260.

Kay, K.M., and Sargent, R.D. (2009). The role of animal pollination in plant speciation: Integrating ecology, geography, and genetics. Annual Review of Ecology, Evolution, and

Klingenberg, C.P. (2016). Size, shape, and form: Concepts of allometry in geometric morphometrics. Development Genes and Evolution 226, 113-137.

Kuhl, F.P., and Giardina, C.R. (1982). Elliptic Fourier features of a closed contour. Computer Graphics and Image Processing 18, 236-258. species of Bolivian Centropogon. American Journal of Botany 106, 633-642.

672 Lagomarsino, L.P., Forrestel, E.J., Muchhala, N., and Davis, C.C. (2017). Repeated evolution of vertebrate pollination syndromes in a recently diverged Andean plant clade. Evolution

Lenth, R., Singmann, H., Love, J., and others (2018). Emmeans: Estimated marginal means, aka least-squares means. R Package Version 1. $32,1229-1234$. 
MacLeod, N. (1999). Generalizing and extending the eigenshape method of shape space visualization and analysis. Paleobiology 25, 107-138.

MacLeod, N. (2002). Geometric morphometrics and geological shape-classification systems. Earth-Science Reviews 59, 27-47.

684 MacLeod, N. (2011). The centre cannot hold I: ZR fourier analysis. Palaeontological Association Newsletter 78, 35-45.

MacLeod, N. (2012). The centre cannot hold II: Elliptic fourier analysis. Palaeontological Association Newsletter 79, 29-42.

MacLeod, N., and Rose, K.D. (1993). Inferring locomotor behavior in paleogene mammals via eigenshape analysis. American Journal of Science 293, 300-355.

Maglianesi, M.A., Blüthgen, N., Böhning-Gaese, K., and Schleuning, M. (2014). Morphological traits determine specialization and resource use in plant-hummingbird networks in the

Maglianesi, M.A., Böhning-Gaese, K., and Schleuning, M. (2015b). Different foraging preferences of hummingbirds on artificial and natural flowers reveal mechanisms structure and specialization in three tropical plant-hummingbird interaction networks

699 Manning, J.C., and Goldblatt, P. (1997). The Moegistorhynchus longirostris (Diptera: 
Martén-Rodríguez, S., Almarales-Castro, A., and Fenster, C.B. (2009). Evaluation of pollination syndromes in Antillean Gesneriaceae: Evidence for bat, hummingbird and generalized flowers. Journal of Ecology 97, 348-359.

Maruyama, P.K., Sonne, J., Vizentin-Bugoni, J., Martín González, A.M., Zanata, T.B., Abrahamczyk, S., Alarcón, R., Araujo, A.C., Araújo, F.P., Baquero, A.C., et al. (2018). Functional diversity mediates macroecological variation in plant-hummingbird interaction networks. Global Ecology and Biogeography 27, 1186-1199.

Mclntyre, G., and Browne, K. (1996). Effect of darkening the cotyledons on the growth and curvature of the sunflower hypocotyl: Evidence of hydraulic signalling. Journal of Experimental Botany 47, 1561-1566.

Miller, E.T., Wagner, S.K., Harmon, L.J., and Ricklefs, R.E. (2017). Radiating despite a lack of character: Ecological divergence among closely related, morphologically similar honeyeaters (Aves: Meliphagidae) co-occurring in arid Australian environments. The American Naturalist 189, E14-E30.

Milnor, J. (1954). On total curvatures of closed space curves. Mathematica Scandinavica 1, 289296.

Minnaar, C., Jager, M. de, and Anderson, B. (2019). Intraspecific divergence in floral-tube length promotes asymmetric pollen movement and reproductive isolation. New Phytologist 1160-1170.

Mountainspring, S. (1987). Ecology, behavior, and conservation of the Maui Parrotbill. The Condor 89, 24-39. 
723 Muller, A. (1995). Morphological specializations in Central European bees for the uptake of

724 pollen from flowers with anthers hidden in narrow corolla tubes (Hymenoptera:

725 Apoidea). Entomologia Generalis 20, 43-57.

726 Navalón, G., Marugán-Lobón, J., Bright, J.A., Cooney, C.R., and Rayfield, E.J. (2020). The

727 consequences of craniofacial integration for the adaptive radiations of Darwin's finches

728 and Hawaiian honeycreepers. Nature Ecology \& Evolution 4, 270-278.

729 Niet, T. van der, and Johnson, S.D. (2012). Phylogenetic evidence for pollinator-driven diversification of angiosperms. Trends in Ecology \& Evolution 27, 353-361.

731 Ollerton, J. (2017). Pollinator diversity: Distribution, ecological function, and conservation.

732 Annual Review of Ecology, Evolution, and Systematics 48, 353-376.

733 Olsen, A.M. (2017). Feeding ecology is the primary driver of beak shape diversification in $734 \quad$ waterfowl. Functional Ecology 31, 1985-1995.

735 Partida-Lara, R., Enriquez, P.L., Vazquez Perez, J.R., and Pineda Diez de Bonilla, E. (2018).

736 Spatio-temporal structure of the taxonomic and functional diversity of hummingbirds at 737 the biosphere reserve El Triunfo, Chiapas, Mexico. Ornitologia Neotropical 29, 37-50.

738 Paton, D., and Collins, B. (1989). Bills and tongues of nectar-feeding birds: A review of morphology, function and performance, with intercontinental comparisons. Australian

$740 \quad$ Journal of Ecology 14, 473-506.

741 Peng, F., Campos, E.O., Sullivan, J.G., Berry, N., Song, B.B., Daniel, T.L., and Bradshaw Jr, H. (2019). Morphospace exploration reveals divergent fitness optima between plants and 743 pollinators. PloS One 14, e0213029. 
744 Pour, A.S., Chegini, G., Zarafshan, P., and Massah, J. (2018). Curvature-based pattern recognition for cultivar classification of Anthurium flowers. Postharvest Biology and Technology 139, 67-74.

R Core Team (2017). R: A language and environment for statistical computing (Vienna, Austria: R Foundation for Statistical Computing).

Raudseps, J.G. (1965). Some aspects of the tangent-angle vs. Arc length representation of contours (Ohio State University Research Foundation, Communication; Control Systems Lab).

Rico-Guevara, A., and Araya-Salas, M. (2014). Bills as daggers? A test for sexually dimorphic weapons in a lekking hummingbird. Behavioral Ecology 26, 21-29.

Ritter, R. (1992). Lateral bending during lizard locomotion. Journal of Experimental Biology $173,1-10$.

Robertson, C. (1889). Flowers and Insects. II. Botanical Gazette 14, 172-178.

757 Rocha, D., Monte Bello, C., Sobol, S., Samach, A., and Dornelas, M. (2015). Auxin and physical constraint exerted by the perianth promote androgynophore bending in Passiflora mucronata L. (Passifloraceae). Plant Biology 17, 639-646.

763 Rohlf, F.J. (2015). The tps series of software. Hystrix 26.

764 Rohlf, F., and Slice, D. (1990). Extensions of the Procrustes method for optimal superposition of landmarks. Systematic Zoology 39, 40-59.

766 Rutter, J.W. (2000). Geometry of Curves (Boca Raton, FL: CRC Press, Taylor; Francis Group). 
767 Schmitt, D., Rose, M.D., Turnquist, J.E., and Lemelin, P. (2005). Role of the prehensile tail during ateline locomotion: Experimental and osteological evidence. American Journal of Physical Anthropology 126, 435-446.

770

Scott-Elliot, G. (1890). Ornithophilous flowers in South Africa. Annals of Botany 4, 265-280.

771 Silk, W.K. (1989). On the curving and twining of stems. Environmental and Experimental Botany 29, 95-109.

Smith, T.B., Freed, L.A., Lepson, J.K., and Carothers, J.H. (1995). Evolutionary consequences of extinctions in populations of a Hawaiian honeycreeper. Conservation Biology 9, 107113.

Snow, B.K., and Snow, D. (1972). Feeding niches of hummingbirds in a Trinidad valley. The Journal of Animal Ecology 471-485. morphologically specialized hummingbirds coincide with floral trait matching across an

Sonne, J., Vizentin-Bugoni, J., Maruyama, P.K., Araujo, A.C., Chávez-González, E., Coelho, Andean elevational gradient. Biotropica 51, 205-218. A.G., Cotton, P.A., Marín-Gómez, O.H., Lara, C., Lasprilla, L.R., et al. (2020).

787 Stearn, W.T. (2002). The genus Epimedium and other herbacious Berberidaceae including the genus Podophyllum (Portland, OR: Timber Press, Inc.). 
Stiles, F.G. (1975). Ecology, flowering phenology, and hummingbird pollination of some Costa Rican Heliconia species. Ecology 56, 285-301.

791 Stiles, F.G. (1995). Behavioral, ecological and morphological correlates of foraging for arthropods by the hummingbirds of a tropical wet forest. Condor 853-878.

793 Stiles, F.G. (2004). Phylogenetic constraints upon morphological and ecological adaptation in hummingbirds (Trochilidae): Why are there no hermits in the paramo. Ornitologia

Stiles, F.G. (2008). Ecomorphology and phylogeny of hummingbirds: Divergence and convergence in adaptations to high elevations. Ornitologia Neotropical 19, 511-519.

798 Temeles, E.J. (1996). A new dimension to hummingbird-flower relationships. Oecologia 105, $517-523$.

800 Temeles, E.J., and Kress, W.J. (2003). Adaptation in a plant-hummingbird association. Science $300,630-633$.

802 Temeles, E.J., Pan, I.L., Brennan, J.L., and Horwitt, J.N. (2000). Evidence for ecological causation of sexual dimorphism in a hummingbird. Science $289,441-443$.

804 Temeles, E.J., Goldman, R.S., and Kudla, A.U. (2005). Foraging and territory economics of sexually dimorphic Purple-Throated Caribs (Eulampis jugularis) on three Heliconia morphs. The Auk 122, 187-204.

807 Temeles, E.J., Koulouris, C.R., Sander, S.E., and Kress, W.J. (2009). Effect of flower shape and size on foraging performance and trade-offs in a tropical hummingbird. Ecology 90, $1147-1161$. 
810 Temeles, E.J., Miller, J.S., and Rifkin, J.L. (2010). Evolution of sexual dimorphism in bill size

811

812

813

814

815

816

817

818

819

820

821

822

823

824

825

826

827

828

829

830

831

832

and shape of hermit hummingbirds (Phaethornithinae): A role for ecological causation.

Philosophical Transactions of the Royal Society B: Biological Sciences 365, 1053-1063.

Terral, J.-F., Alonso, N., Capdevila, R.B.i., Chatti, N., Fabre, L., Fiorentino, G., Marinval, P., Jordá, G.P., Pradat, B., and Rovira, N. (2004). Historical biogeography of olive domestication (Olea europaea L.) As revealed by geometrical morphometry applied to biological and archaeological material. Journal of Biogeography 31, 63-77.

Travers, S.E., Temeles, E.J., and Pan, I. (2003). The relationship between nectar spur curvature in jewelweed (Impatiens capensis) and pollen removal by hummingbird pollinators. Canadian Journal of Botany 81, 164-170.

Tripp, E.A., and McDade, L.A. (2013). Time-calibrated phylogenies of hummingbirds and hummingbird-pollinated plants reject a hypothesis of diffuse co-evolution. Journal of Systematic and Evolutionary Botany 31, 89-103.

Vamosi, J.C., Magallón, S., Mayrose, I., Otto, S.P., and Sauquet, H. (2018). Macroevolutionary patterns of flowering plant speciation and extinction. Annual Review of Plant Biology 69, 685-706.

Van Otterloo, P.J. (1991). A contour-oriented approach to shape analysis (Prentice Hall New York, NY).

Wang, Q., Li, Y., Pu, X., Zhu, L., Tang, Z., and Liu, Q. (2013). Pollinators and nectar robbers cause directional selection for large spur circle in Impatiens oxyanthera (Balsaminaceae). Plant Systematics and Evolution 299, 1263-1274.

Webster, M., and Sheets, H.D. (2010). A practical introduction to landmark-based geometric morphometrics. The Paleontological Society Papers 16, 163-188. 
833 Weinstein, B.G., and Graham, C.H. (2017). Persistent bill and corolla matching despite shifting 834 temporal resources in tropical hummingbird-plant interactions. Ecology Letters 20, 326-

$835 \quad 335$.

836 Wood, S.N. (2017). Generalized additive models: An introduction with r (Boca Raton, FL: CRC

837 Press).

838 Young, H.J. (2008). Selection on spur shape in Impatiens capensis. Oecologia 156, 535-543.

839 Zahn, C.T., and Roskies, R.Z. (1972). Fourier descriptors for plane closed curves. IEEE

$840 \quad$ Transactions on Computers 100, 269-281.

841 Zwillinger, D. (2018). In CRC Standard Mathematical Tables and Formulae, (Boca Raton, FL:

842 Chapman; Hall, CRC Press), pp. 424-425. 